\title{
Relationships between Plasmodium falciparum infection and morbidity in a highly endemic area
}

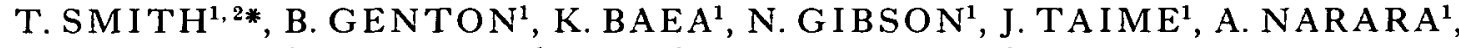 \\ F. AL-YAMAN $N^{1}$, H.-P. BECK $K^{1}, J$. HII I $I^{1}$ and M. ALPERS ${ }^{1}$ \\ ${ }^{1}$ Papua New Guinea Institute of Medical Research, P.O. Box 378, Madang, and P.O. Box 60, Goroka, \\ Papua New Guinea \\ ${ }^{2}$ Swiss Tropical Institute, Socinstrasse 57, Postfach, 4002 Basel, Switzerland
}

(Received 24 January 1994; revised 28 April 1994; accepted 28 April 1994)

SUMMARY

A total of 736 outpatients diagnosed as having malaria using clinical criteria at a health centre in a highly endemic area of Papua New Guinea were investigated parasitologically. Plasmodium falciparum-attributable fractions were determined using a logistic regression model to compare parasite densities in cases with those of healthy individuals in community surveys. Thirty-seven percent of presumptive cases were found to have raised $P$. falciparum parasitaemia. This corresponds to an average reporting rate for the population of 0.53 attributable episodes per annum. Whilst the maximum prevalence of parasitaemia in the community was in children aged 5-9 years, the maximum age-specific incidence of attributable cases at the outpatient clinic was 2 cases per annum in the 2- to 4-year-old age group. The procedure for estimating attributable fractions makes it possible to compare morbidity rates between age groups, and to examine how the relationship between morbidity risk and parasite density changes with age, without diagnosing individual episodes. The average tolerance of parasites in an age group was measured by considering the level of parasitaemia associated with a given risk of malaria-attributable morbidity. In contrast to anti-parasite immunity, tolerance of parasites declines with age since at parasite isodensity the probability of being symptomatic increases with age.

Key words: malaria, epidemiology, diagnosis, parasitaemia, Papua New Guinea.

\section{INTRODUCTION}

The precise magnitude of the public health problem posed by malaria has always been unclear in highly endemic areas since many people have parasites in the blood without any symptoms or signs of disease (Greenwood, 1987). In such areas a diagnosis of malaria cannot be made with certainty, even when slide-readings are available. It has also been unclear how cases should be defined for epidemiological purposes. Different case definitions have been used in field or hospital-based studies (e.g. Greenwood et al. 1987; Trape et al. 1985, 1987; Snow et al. 1988; Olivar et al. 1991) and since there is little standardization it has been difficult to compare the results of different studies.

Many definitions require a malaria case to have parasitaemia exceeding a given threshold. If the threshold is chosen arbitrarily there is likely to be over- or underestimation of the number of malaria attributable cases (bias). However, the relationship between illness and parasitaemia depends on the

* Address for correspondence: Dr T. Smith, Department of Public Health and Epidemiology, Swiss Tropical Institute, Socinstrasse 57, Postfach, CH-4002 Basel, Switzerland.

+ Present address: Department of Public Health and Tropical Medicine, James Cook University, Townsville, QLD, Australia. immune status of the host. The extent of this bias will therefore depend both on ages of the patients and on malaria exposure. Endemicity varies from area to area and from time to time. Therefore it can be difficult to interpret comparisons of the incidence of malaria morbidity between areas or between age groups even when the same case definition is used throughout.

Recent analyses of data from a highly endemic area of Tanzania used a logistic regression method to compare the parasite densities of febrile children in the community with those of afebrile controls in order to estimate the risk of fever as a continuous function of parasite density. This was used to determine what proportion of the febrile children have Plasmodium falciparum parasitaemia raised above the level in the afebrile children, and hence an illness that can be attributed to malaria (Smith et al. 1994). In this report we use the same method to look at the relationship between parasitaemia and morbidity over the whole age range in a highly endemic area of Papua New Guinea. Instead of febrile cases from community surveys, presumptive cases of malaria in a health facility were analysed.

Investigations of presumptive malaria cases at the Kunjingini health centre in the East Sepik Province of Papua New Guinea have been carried out since late 1990 as part of the Malaria Vaccine Epidemiology and Evaluation Project (MVEEP) (Alpers et 
al. 1992) with the objective of evaluating measures of malaria-specific morbidity. The investigations carried out include the preparation of thick and thin blood smears for parasitological examination. During the same period, community malariological surveys were carried out in several of the villages served by the health centre.

By applying the method of data analysis of Smith et al. (1994) to the case-control data from the MVEEP we have been able to examine the age dependence of the relationship between morbidity risk and parasite density, and hence of $P$. falciparum malaria-attributable morbidity in the health centre cases investigated. These results are used to examine the age-dependence of immunological tolerance and of anti-parasite immunity.

\section{MATERIALS AND METHODS}

\section{Study area and population}

The study area was the Wosera, in the Maprik district in East Sepik Province of Papua New Guinea with a population of 22587 persons recorded at the national census in 1990 . Whilst communications and infrastructure of health services are relatively good, indices of health are poor and there is relatively little socio-economic development (Garner, 1989). The area is highly endemic for malaria with the following parasite formula: $0.55 ; 0.25 ; 0.2(\mathrm{Pf} ; \mathrm{Pv} ; \mathrm{Pm})$ (Genton et al. 1994). There is little seasonal variation in rainfall and malaria transmission is perennial.

There are three health centres in the Wosera area, each providing primary health care for a distinct group of villages. The village aid-post system is not very effective. There are, however, a number of practising traditional healers.

\section{Demographic surveillance}

For the purpose of the MVEEP, demographic and epidemiological surveillance is carried out in 10 villages selected for their different levels of malarial endemicity, with a total population of approximately 4000. A complete census of these villages is carried out annually. In each village, one or more villagers are employed to record vital events, including pregnancies, births, deaths and migrations in or out of the village.

Five of these villages fall in the catchment area of the Kunjingini Health Centre. This paper considers data only for these five villages.

\section{Community surveys}

Two cross-sectional malariological surveys were carried out, one in April-May 1991 and the other in August-September 1991. The target for each survey was to include at least one third of the households in the village, selected randomly. Thick and thin blood films were prepared from all consenting individuals in these households. Morbidity questionnaires were also administered, during which respondents (or their guardians in the case of children) were asked if any illness had been experienced in the previous week.

\section{Kunjingini Sub Health Centre (KHC) surveillance}

Kunjingini Sub Health Centre (KHC) is a missionrun health facility located in Kunjingini-1 village. All new outpatients were recorded at KHC with details of age, sex, place of residence, presumptive diagnosis and treatment. Patients returning to complete a previously prescribed course of treatment were not recorded.

The nurse staffing the outpatient clinic carried out diagnoses and prescribed treatments. Diagnostic procedures at $\mathrm{KHC}$ follow the guidelines of the Papua New Guinea Department of Health, which recommend malaria treatment for all patients with fever (Papua New Guinea Department of Health, 1989; Vince, 1992). In most cases, diagnosis of malaria was on the basis of a history of fever without any other major symptom or sign.

A member of the Institute of Medical Research (IMR) staff attended the outpatient clinic where possible and carried out further investigations on an unselected sample of the presumptive malaria cases. Data collected include a detailed history, a clinical examination, haemoglobin and packed cell volume determination (Genton et al. 1994). Thick and thin films were again prepared for parasitological assessment.

\section{Laboratory methods}

A thick smear and a thin smear were made from each blood sample. Slides were stained using $4 \%$ Giemsa and the malaria parasite species and density assessed by counting parasites against 200 leucocytes. Parasite counts were converted to numbers of parasites $/ \mu$ by assuming a standard leucocyte count of $8000 / \mu \mathrm{l}$ (Cattani et al. 1986; Shute, 1988). In this study, only $P$. falciparum parasitaemias are considered.

Blood films were examined by the same team of microscopists, irrespective of whether they came from the community surveys or the health centre surveillance.

\section{Case and control definitions}

Estimates of attributable fractions were made using the data for the presumptive cases from which blood films had been collected. Control blood films were those collected in community surveys from persons who did not report illness during the previous week. Only control data from the 5 villages in the catchment area of the health centre were included in the present analyses. 


\section{Attributable fraction estimates}

The proportion of cases in which the illness was associated with raised $P$. falciparum parasitaemia is called the attributable fraction $(\lambda)$. Two methods were used to estimate attributable fractions.

(1) The attributable fraction $(\lambda)$ was estimated by logistic regression as described by Smith et al. (1994).

The dataset analysed consists of parasite densities measured on cases and also on healthy community controls. Each case, or control, is indexed by a variable $i$. Let $N$ be the total number of cases, and $R_{i}$ be the ratio of the odds of being a case associated with individual $i$ with parasite density $x_{i}$, compared with a corresponding individual without parasites; $\lambda$ is then estimated as the mean of the excess risk associated with the different cases, i.e.

$\lambda=\frac{1}{N} \sum_{i} \frac{R_{i}-1}{R_{i}}$,

where the summation is over the cases only. Similarly, the quantity

$\lambda_{i}=\frac{R_{i}-1}{R_{i}}$

gives an estimate of the probability that case $i$ is malaria attributable.

The basic logistic regression model used to estimate the values of $R_{t}$ was

$\log \frac{p_{i}}{1-p_{i}}=\beta_{\mathrm{o}}+\beta_{1} x_{i}^{\tau}$

where $p_{i}$ is the fitted probability that individual $i$ is a case rather than a community control and $\beta_{0}, \beta_{1}$ and $\tau$ are parameters to be estimated.

$R_{i}$ and hence $\lambda_{i}$, are then estimated using

$R_{i}=\exp \left(\beta_{1} x_{i}^{T}\right)$.

Bruzzi et al. (1985) have shown that attributable fractions estimated in a similar way from logistic regression models can be adjusted for covariate dependences by including extra terms in the model. By fitting additional linear terms in the model (3) we tested whether the intercept, $\beta_{0}$ depended on village of origin, age or time of year. Statistical significance was tested using likelihood ratio tests. Ages were grouped as shown in Table 1 . Analysis by time of year grouped each case with control data taken from the field survey closest in time to the date of presentation of the case.

Further models were fitted to test whether $\beta_{1}$ depended upon these covariates. A final model was selected which incorporated important covariate dependences of $\beta_{0}$ and $\beta_{1}$ and hence gave the best estimates of $R_{i}$.

(2) The parasite prevalences in presumptive malaria cases $\left(p_{1}\right)$ were compared with those in com-

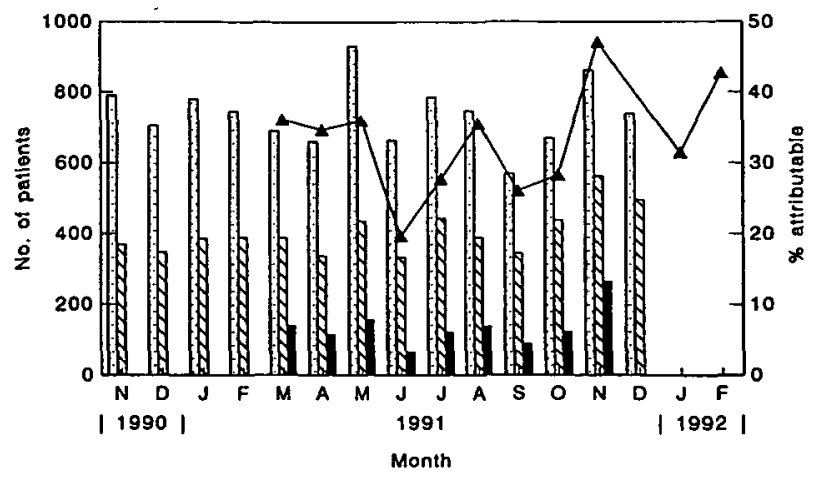

Fig. 1. Numbers of patients from 5 study villages reporting to Kunjingini Health centre, by month.

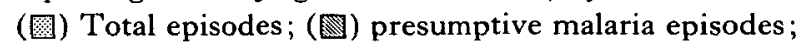

(D) Plasmodium falciparum-attributable episodes. The line indicates the $P$. falciparum-attributable fraction by month.

munity controls $\left(p_{0}\right)$. The proportion of cases which had parasitaemias unrelated to the cause of their illness was estimated by assuming the parasite prevalence in these patients to be the same as that in the controls. The proportion of cases attributable to parasitaemia could then be estimated as $\left(p_{1}-p_{0}\right) /$ $\left(1-p_{0}\right)$. This estimate was not adjusted for covariates, but separate estimates were made for different age groups.

\section{Age-specific relative risks}

Age-specific relative risks were computed for (i) total consultations at the health centre, (ii) consultations with presumptive malaria, and (iii) $P$. falciparumattributable episodes. The morbidity risk (episodes/ inhabitant) in each group was divided by the risk in the youngest age group to get the risk relative to the under-2-year age group. The numbers of inhabitants were taken from the demographic surveillance system.

The same level of parasitaemia might be associated with different morbidity risks in different age groups. In order to investigate this, the risk of a $P$. falciparum-attributable consultation at the clinic was computed as a function of the level of parasitaemia $(x)$, and the age group $(a)$, relative to the under-2year age group $(a=0)$ and a standard level of parasitaemia (10000 parasites $/ \mu \mathrm{l})$. The details of how this was computed are presented in the Appendix.

\section{RESULTS}

\section{Sample sizes}

The resident population was 1602 on 1 July 1991 . An annual total of 3995 visits to the outpatient clinic ( $2 \cdot 5$ per person) was recorded. Fig. 1 shows how the numbers of patients varied by month: there was negligible seasonality evident and $58 \%$ of these visits were recorded as presumptive malaria. 
Table 1. Attendances and positivity of blood slides by age group

\begin{tabular}{|c|c|c|c|c|c|c|c|}
\hline \multirow[b]{2}{*}{$\begin{array}{l}\text { Age group } \\
\text { (years) }\end{array}$} & \multirow[b]{2}{*}{$\begin{array}{l}\text { Total } \\
\text { population* }\end{array}$} & \multirow[b]{2}{*}{$\begin{array}{l}\text { Community } \\
\text { blood slides } \dagger\end{array}$} & \multirow[b]{2}{*}{$P_{p f}+\mathrm{g}$} & \multicolumn{3}{|c|}{ Health centre attendances } & \multirow[b]{2}{*}{$P_{p f} \S \Phi$} \\
\hline & & & & Totalf & $\begin{array}{l}\text { Presumptive } \\
\text { malariaf }(\%)\end{array}$ & $\begin{array}{l}\text { Blood } \\
\text { slides§ }\end{array}$ & \\
\hline $0-1$ & 175 & 99 & $12 \cdot 1$ & 1174 & $59 \cdot 5$ & 150 & $27 \cdot 3$ \\
\hline $2-4$ & 152 & 190 & $34 \cdot 2$ & 800 & $76 \cdot 6$ & 197 & $66 \cdot 0$ \\
\hline $5-9$ & 242 & 283 & $46 \cdot 3$ & 564 & $64 \cdot 2$ & 138 & $68 \cdot 1$ \\
\hline $10-19$ & 355 & 377 & $40 \cdot 9$ & 500 & $48 \cdot 6$ & 85 & $57 \cdot 7$ \\
\hline $20-39$ & 433 & 472 & $25 \cdot 0$ & 526 & $47 \cdot 9$ & 113 & $43 \cdot 4$ \\
\hline $40+$ & 245 & 287 & $18 \cdot 5$ & 431 & $36 \cdot 0$ & 53 & $30 \cdot 2$ \\
\hline Total & 1602 & 1708 & $31 \cdot 2$ & 3995 & $58 \cdot 2$ & 736 & $51 \cdot 5$ \\
\hline
\end{tabular}

* De jure population of the 5 study villages on 1 July 1991

+ Slides from health individuals surveyed in the community during the 2 control surveys.

$\ddagger$ Health centre attendances during the 1 year period Jan 1991-Dec 1991.

$\S$ Blood slides prepared from presumptive malaria cases at the health centre during the 1 year period March 1991Feb 1992.

I $P_{p f}$ is the percentage of slides positive for Plasmodium falciparum.

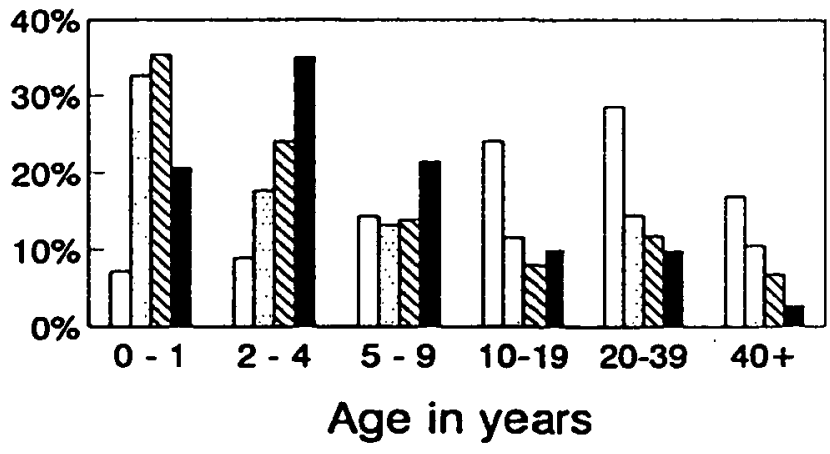

Fig. 2. Percentage of patients reporting to Kunjingini Health Centre, in different age groups. ( $\square$ ) Censused population; (圈) all episodes; ( $\mathbb{Q}$ ) presumptive malaria episodes; ( $\square$ ) Plasmodium falciparum-attributable episodes.

In both community surveys, well over the target one third of the population were seen, 782 individuals being seen during the survey in April-May 1991. Of these, 102 reported some illness in the previous week and are not considered in the present analyses. Blood films for parasitological analysis were prepared from 675 of the remainder. During the August-September survey, only 80 individuals out of 1148 seen were excluded because of illness. A total of 1033 blood films was prepared from healthy individuals.

\section{Age distributions}

Table 1 shows the age breakdowns of the population as recorded by the demographic surveillance, of the health centre patients, and of the individuals bled at the community surveys. Most of the patients at the outpatient clinic were children (Fig. 2) and the relative risk of attendance (allowing for the population age distribution) was even more age dependent (Fig. 3), with a steep decline in attendance rate with age. The proportion of presumptive malaria patients who were under 10 years old was even higher than the overall proportion of patients (Fig. 2). In contrast, the youngest age group was somewhat under-represented in the community surveys because of the reluctance of parents to allow very small children to be bled, but otherwise the age distributions of the survey participants and the general population were similar.

\section{Parasite prevalence and density}

A greater proportion of the slides prepared at the health centre was positive for $P$. falciparum, than those prepared in the field surveys. This was true for all age groups (Table 1). The highest prevalence was in the 5-9 year age group, both in the patients and in the field surveys.

Fig. 4 shows cumulative parasite density distributions by age. Note that a distribution with a high average density appears as a line towards the bottom of the chart, whilst a distribution with a low density appears as a line towards the top. Fig. 4A illustrates the cumulative parasite density distributions in community controls by age group. In all age groups, very high parasitaemias were unusual in controls. However, there were clear differences at lower parasite densities where the highest parasite densities were found in the 5-9 year age group. The $0-1$ age group was exceptional in including both the highest proportion of aparasitaemic samples and also a relatively high proportion of moderately high parasitaemia samples.

Fig. 4B shows the corresponding cumulative parasite density distributions for presumptive malaria cases. There was much more variation between the age groups than among the controls. Again, the youngest age group shows both the highest proportion of aparasitaemic individuals, but also a 


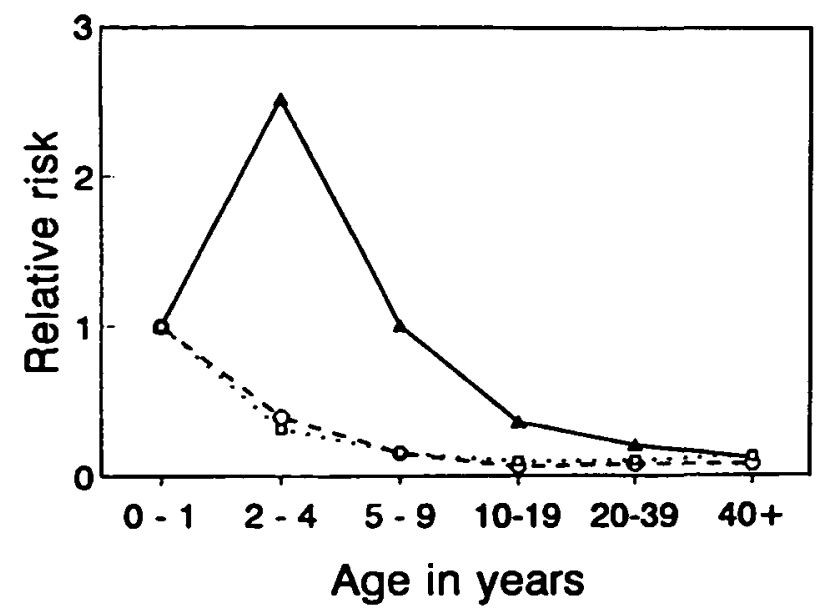

Fig. 3. Risk of morbidity at Kunjingini Health Centre by age group, relative to the youngest age group.

( $\square$ ) All episodes; (O) presumptive malaria episodes;

(A) Plasmodium falciparum-attributable episodes.
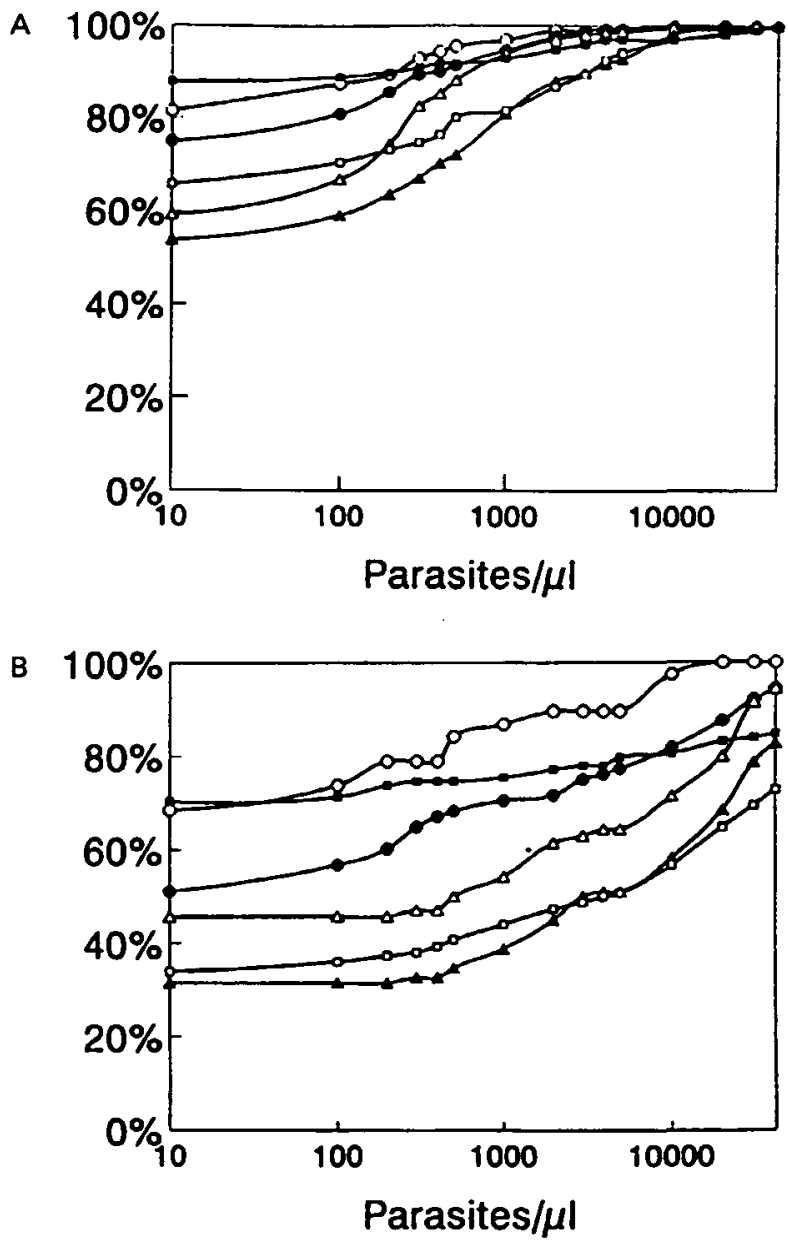

Fig. 4. Cumulative distribution of parasite densities by age group. (A) Community surveys; (B) presumptive malaria cases. Age groups (years): (D) $0-1$; (口) 2-4;

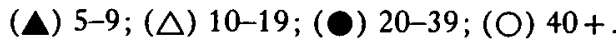

relatively high proportion of very high density cases. In other age groups, a high prevalence was associated with a high frequency of high density cases, with the highest densities in the 2-4 and 5-9 age groups.
There was very little difference between the two community surveys in the distributions of parasitaemia, or in the parasitaemias of the health centre cases during different times of the year. In the field surveys, Nale village had fewer aparasitaemic individuals than the other villages. Kunjingini- 1 had the highest prevalence and density among presumptive cases.

\section{Logistic regression models}

The results of the logistic regression analysis of risk factors for becoming a presumptive case are shown in Table 2. The analysis included two sets of terms. Firstly, it was necessary to include model terms to allow for factors which affected the relative risk of becoming a presumptive malaria case, but not necessarily the risk that the morbidity was actually malaria associated. These terms constitute the main effects in the logistic regression model. Because more individuals attended the second field survey than the first, the time-period affected the relative risk of being a case (defined by the field survey with which the case or control was associated), and a term in time-period was therefore included in the model. Since age affects both the morbidity risk and also the chance that an individual was sampled in a field survey, terms in age group were also included in the model and proved highly statistically significant. Attendance at the health centre is also likely to be affected by the difficulty of reaching the facility which reflects the proximity of an individual's home, therefore variables indicating the village of origin of the case (or control) were included in the model.

The second group of terms in the logistic regression analysis were those which estimated the relationship between risk and parasite density. Preliminary analyses were carried out to determine the best-fitting value of the exponent $\tau$. A value of 0.84 was chosen. Subsequent analyses confirmed the general impressions gained by comparing parasite density distributions. There was no difference between the time-periods in the risk associated with a specific level of parasitaemia. The interaction with time-period was therefore not included in the final model. There were, however, substantial differences between age groups in the best-fitting value of the regression coefficient $\beta_{1}$, and the regression coefficient for Kunjingini-1 village was substantially higher than for the other villages, which were comparable with each other in this respect.

Fig. 5 shows the fit of the final logistic regression model to the data. In order to make it possible to plot out observed values of $p_{i}$, the data were grouped into parasitaemia classes. The mean observed value of $p_{i}$ for each class was then plotted against parasite density. Over most of the parasitaemia range, there was a gradual increase in $p_{i}$ with increasing parasitaemia; however, there was a decrease between 0 
Table 2. Logistic regression analysis of factors affecting the risk of presumptive malaria at Kunjingini Health Centre

\begin{tabular}{llcc}
\hline \hline Effect & $\begin{array}{l}\text { Degrees } \\
\text { of freedom }\end{array}$ & $\begin{array}{l}\text { Likelihood } \\
\text { ratio } \chi^{2}\end{array}$ & $P$ value \\
\hline Main effects & & & \\
$\quad P$. falciparum parasitaemia & 1 & $366 \cdot 5$ & $<0.00001$ \\
Time-period & 1 & $10 \cdot 7$ & 0.001 \\
Age group & 5 & $146 \cdot 2$ & $<0.00001$ \\
$\quad$ Kunjingini-1 & 1 & $0 \cdot 03$ & 0.86 \\
$\quad$ Other villages & 3 & $4 \cdot 72$ & 0.19 \\
All villages & 4 & $4 \cdot 75$ & 0.31 \\
Interactions with & & & \\
parasitaemia effect & & & \\
Time-period & 1 & 0.05 & 0.82 \\
Age group & 5 & 20.5 & 0.00001 \\
$\quad$ Kunjingini-1 & 1 & 6.1 & 0.014 \\
$\quad$ Other villages & 3 & $5 \cdot 6$ & 0.13 \\
All villages & 4 & 11.7 & 0.019 \\
\hline \hline
\end{tabular}

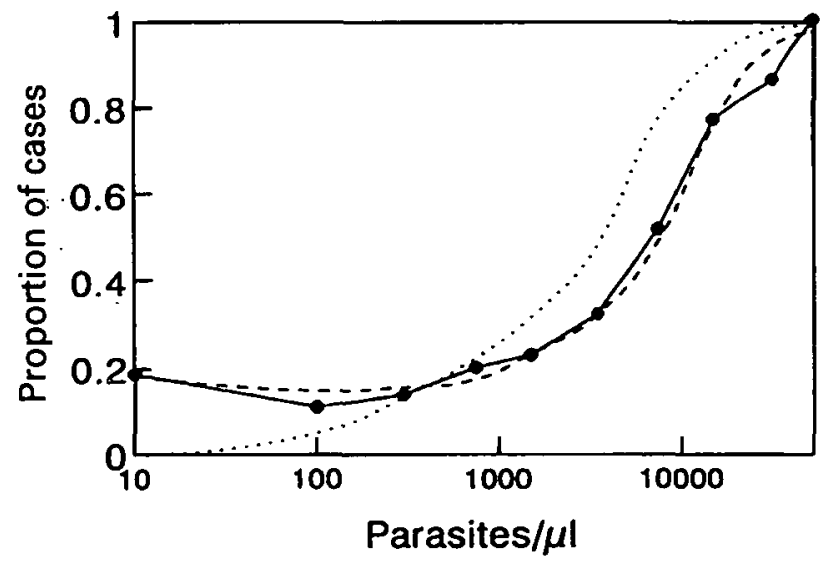

Fig. 5. Proportion of cases among cases and controls with given parasite densities. (O) Observed proportion; (---) fitted proportion from logistic regression model; (....) Plasmodium falciparum-attributable fraction.

parasites $/ \mu 1$ and 100 parasites $/ \mu 1$. The mean fitted values of $p_{i}$ from the regression model are also shown, and fit well over most of the curve. The initial decrease is not, however, modelled very closely. Corresponding to the fitted curve for $p_{i}$ is the curve of how $\lambda_{i}$ depends on parasitaemia. This shows a smooth increase over the range of parasitaemias.

\section{Attributable fractions and attributable morbidity}

Table 3 gives the algorithm for estimating the probability that an individual case is malaria attributable from the logistic regression results. This method was used to obtain the estimates used in Figs $1-3$, and in Table 4 . These logistic regression estimates are higher than AF estimates obtained by comparing unadjusted parasite prevalences (Table 4 ), in all except the oldest age group.

The overall proportion of the presumptive cases which is attributable was estimated as 0.366 using
Table 3. Parameter estimates used to estimate attributable fractions

(The relative risk of illness, for individual $i$ in age-group $j$ and parasitaemia $x$ relative to an equivalent aparasitaemic individual is estimated as $R_{t}=\exp \left(10^{-3} \beta, x^{T}\right)$, where $\tau$ is 0.84 and $\beta$, is read from Table 3 , for all villages other than Kunjingini-1. For patients from Kunjingini-1 a further term of 0.409 (s.E. 0.189 ) was added to $\beta_{1}$ before computing R.)

\begin{tabular}{llll}
\hline \hline $\begin{array}{l}\text { Age group } \\
\text { (years) }\end{array}$ & $j$ & $\begin{array}{l}\text { Parameter } \\
\text { est. }(\beta,)\end{array}$ & $\begin{array}{l}\text { Standard } \\
\text { error }\end{array}$ \\
\hline $0-1$ & 1 & $0 \cdot 325$ & $0 \cdot 120$ \\
$2-4$ & 2 & 0.593 & $0 \cdot 102$ \\
$5-9$ & 3 & 0.720 & $0 \cdot 126$ \\
$10-19$ & 4 & $1 \cdot 20$ & $0 \cdot 232$ \\
$20-39$ & 5 & 1.08 & 0.268 \\
$40+$ & 6 & 1.41 & 0.484 \\
\hline \hline
\end{tabular}

the logistic regression approach, with the attributable fraction among the presumptive cases highest in the 2-4 year age group and lowest in the oldest groups (Table 4). The corresponding annual incidences of attributable cases at the health centre are also shown in Table 4 . Fevers among adult are very unlikely to be associated with raised parasitaemia in this population, whilst in infants, there are many more non-malarial fevers than in older individuals. Thus, although the age-specific relative risk of malaria-attributable morbidity shows a decline with age in the older age groups, the maximum age-specific relative risk is in the $2-4$ year age group (Fig. 3), as is the maximum proportion of malaria-attributable cases (Fig. 2).

The estimated risk of malaria-attributable morbidity associated with given levels of parasitaemia increased both with the level of parasitaemia, and perhaps surprisingly, with age (Fig. 6). Thus the 
Table 4. Attributable fraction estimates and annual incidence of attributable cases

\begin{tabular}{|c|c|c|c|}
\hline \multirow{2}{*}{$\begin{array}{l}\text { Age group } \\
\text { (years) }\end{array}$} & \multicolumn{2}{|c|}{$\begin{array}{l}\text { Attributable } \\
\text { fractions }\end{array}$} & \multirow{2}{*}{$\begin{array}{l}\text { Attributable } \\
\text { episodes/ } \\
\text { year/person* }\end{array}$} \\
\hline & $\lambda *$ & $\lambda \dagger$ & \\
\hline $0-1$ & 0.195 & 0.173 & 0.78 \\
\hline $2-4$ & 0.487 & 0.483 & 1.96 \\
\hline $5-9$ & 0.519 & 0.406 & 0.78 \\
\hline $10-19$ & 0.415 & 0.284 & 0.28 \\
\hline $20-39$ & 0.278 & 0.245 & $0 \cdot 16$ \\
\hline $40+$ & 0.136 & 0.144 & 0.09 \\
\hline All & 0.366 & 0.295 & 0.53 \\
\hline
\end{tabular}

* Attributable fraction estimated as described in Table 3. $\dagger$ Attributable fraction estimated as $\left(p_{1}-p_{0}\right) /\left(1-p_{0}\right)$, not adjusted for covariates.

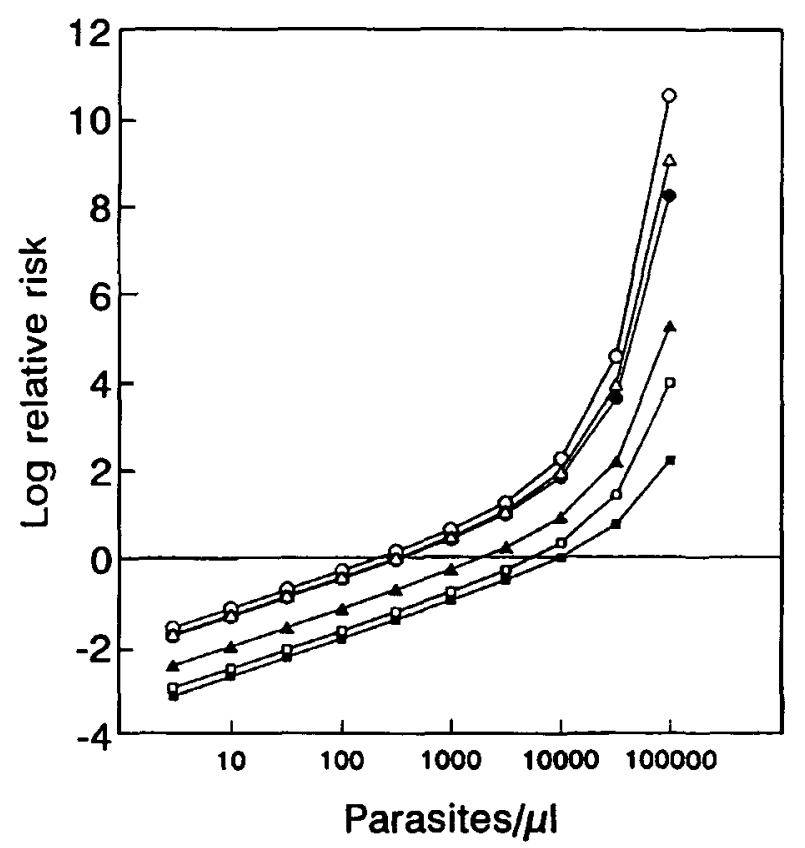

Fig. 6. Risk of reporting to Kunjingini Health Centre with a malaria-attributable episode. Relative risks are broken down by age group and parasite density. Age

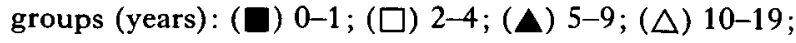
(๑) $20-39 ;(\bigcirc) 40+$. Risks are relative to the risk in the youngest age group at a parasite density of $10^{4}$ parasites $/ \mu 1$.

decline in malaria morbidity with age results entirely from the reduced frequency of high levels of parasitaemia in older individuals.

\section{DISCUSSION}

Malariometric indices such as parasite rates and spleen rates have been used extensively in the past to assess the impact of interventions against malaria, but it is not known whether these indices have a straightforward relationship with the extent of the health problem posed by malaria (Marsh, 1992). As emphasis has shifted from eradication campaigns to malaria control the quantification of malaria morbidity has become more important and it has become apparent that measures of the frequency of clinical disease in endemic areas are inadequate. The number of presumptive cases of malaria diagnosed by the usual staff is a good indicator of the anti-malarial drug requirements but depends very much on diagnostic idiosyncrasies of nurses and clinicians, even in a situation such as that of Papua New Guinea where a fair degree of standardization of diagnostic procedures has been achieved. In order to have a more accurate estimate of incidence, we need to know what proportion of these cases are actually malaria. In this paper we have shown how this proportion can be estimated even when individual cases cannot be diagnosed with certainty.

The number of attributable cases per head of population reporting to a well-used health facility gives a measure of the amount of malaria morbidity potentially faced by the health services. Passive case detection within health services also provides potential outcome measures for randomized trials of interventions. Estimates of the numbers of attributable cases can be used to estimate the required sample size and power of such trials. However, people do not always use the health facility when they are sick, therefore the number of cases at the health centre will always underestimate the total number of cases in the community.

In this study we compared estimates of attributable fractions obtained by two different statistical procedures. The estimates obtained from $2 \times 2$ tables are generally lower than those estimated by logistic regression. The same result was found in the Tanzanian and Gambian studies (Smith et al. 1994; Armstrong Schellenberg et al. 1994), where it was argued that this is likely to be because parasitaemias are reduced in non-malaria cases as a result of the inhibitory effect of fever on the development of P. falciparum (Kwiatkowski, 1989, 1991). Low parasitaemia is associated with a reduced risk of morbidity when compared to no parasitaemia at all. In addition anti-parasite effects of non-specific immune responses to non-pyrogenic infectious agents (Rooth \& Bjorkmann, 1992) would have the same effect in this study. Such effects would bias the estimates from the $2 \times 2$ table method.

An additional advantage of the logistic regression method is that it does not depend on the sensitivity of the detection of parasites. Polymerase chain reaction (PCR) techniques have demonstrated that optical microscopy underestimates the true malaria infection prevalence in the Wosera (Felger et al. 1994). Improved sensitivity in detecting parasites might well affect attributable fraction estimates made by the $2 \times 2$ table method. For these reasons, we consider the attributable fraction estimates obtained using the logistic regression approach to be pref- 
erable to those obtained from $2 \times 2$ tables. Inclusive criteria are used for diagnosing presumptive cases in Papua New Guinea, so few true malaria cases should be excluded and multiplication of the attributable fraction by the number of presumptive cases should give a good estimate of the true total of $P$. falciparum cases.

The logistic regression estimates imply that each individual in the catchment of the Kunjingini Health Centre reports to the health centre with a $P$. falciparum-attributable episode an average of 0.53 times per year. In the case of children in the 2-4 year age range, the attendance rate is almost two $P$. falciparum-attributable episodes per annum. These estimates are plausible and are consistent with other studies (including that of Cox et al. (1994) in Papua New Guinea) which concluded that raised parasitaemia is characteristic of symptomatic malaria episodes. In addition, $P$. vivax causes morbidity in the area and $P$. malariae is also found.

In highly endemic areas, $P$. falciparum parasite rates normally reach a peak in children under 5 years of age and decline substantially later, indicating increasing protection from acquired immunity (Wilson, Garnham \& Swellengrebel, 1950). Where the endemicity is lower, the peak prevalence is in older individuals (Molineaux, 1988). In the Wosera $P$. falciparum prevalence peaks in the 5-9 year age group as in the comparable area of Madang (Cattani et al. 1986; Cox et al. 1994). This is despite relatively high inoculation rates, at least in adults in the latter area (Burkot et al. 1987). The unusual age-prevalence may be a consequence of the biting cycles and activity patterns of the vectors in PNG, predominantly members of the Anopheles punctulatus complex. These differ from those of the $A$. gambiae complex responsible for most perennial transmission in Africa (Charlwood, Graves \& Alpers, 1986). Alternatively, the age-prevalence might be explicable as a result of the very high anti-malarial consumption in the children under 5 years old.

Whatever the reason for this delay in the acquisition of immunity to blood-stage parasites, one effect is to make it possible to look in some detail at the temporal relationship between the acquisition of physiological tolerance and of anti-parasite immunity, and to examine how malaria morbidity is related to these. A couple of notes of caution are needed in interpreting these aspects of our results. Firstly, we used the same estimated leucocyte count for each age group. Since actual leucocyte counts show age dependence, our results should strictly be seen in terms of age dependence of effects associated with parasite-leucocyte ratios rather than parasites per unit of blood volume. Secondly, we do not make any allowance for age dependence in treatment seeking behaviour. However, the differences between age groups are so large that we do not think that these effects can account for them.
Data on the age-dependence of malaria morbidity are difficult to interpret where the problem of distinguishing malaria cases from non-malarial fevers has not been satisfactorily resolved. Overall fever risks frequently show the monotonic decline with age seen in presumptive malaria cases in Kunjingini. This is the typical age dependence pattern for the incidence of lower respiratory tract infections in PNG (Selwyn, 1990; Smith et al. 1991) which undoubtedly form a large proportion of these cases. The estimates of attributable fractions clearly show that the age-prevalence for $P$. falciparum morbidity is quite different, with the peak incidence at the health centre in the 2-4 year age group, younger than the age group with the maximum parasite prevalence.

If a standard parasite density cutoff had been used to define $P$. falciparum morbidity, this too would have given a different pattern of age dependence from that determined using the attributable fraction estimates, since we find that the regression line relating morbidity risk to parasite density becomes steeper as individuals get older and the absolute risk of being ill with a given parasite density is higher in older individuals.

This has implications for our understanding of the development of immunity to malaria. This immunity affects both the density of parasites (anti-parasite immunity) and the risk and extent of morbidity associated with a given parasite density (anti-disease immunity or physiological tolerance). The increase in the gradient of the regression line with age implies that tolerance of parasites declines with age. It is well known that anti-disease immunity rises rapidly in infants (McGregor, 1983). It has also become apparent that this tolerance of malaria parasites is closely related to a loss of sensitivity to tumour necrosis factor (reviewed by Clark \& Chaudhri, 1989). When induced in animal models, this tolerance of 'malaria toxins' is a 'T-independent phenomenon with the response mainly comprising transient IgM antibodies (Bate et al. 1990). If the same applies in humans, then a reduction of toxin due to a reduction of parasite numbers would then in turn reduce this $\mathrm{T}$-cell independent stimulation and anti-disease immunity would wane with increasing anti-parasite immunity (for review see Playfair et al. 1990). Since high parasitaemia is less frequent in older individuals, this hypothesis would indeed predict reduced tolerance in adults.

The transience of tolerance can explain why individuals from high endemicity areas who spend time in malaria-free areas rapidly lose immunity and become ill when re-exposed. Levels of some antibodies and cellular responses which may form part of anti-parasite immunity are also known to fall in semi-immune individuals who experience a period without exposure (Deloron et al. 1987; Chougnet, Deloron \& Savel, 1991). These phenomena might 
account for anomalous behaviour of Kunjingini-1 with higher prevalence and a steeper regression of morbidity risk on parasite density than other villages. The population of this village includes mission employees who are more mobile than those of the other villages, and since the health facility is immediately accessible they are more likely to be treated with anti-malarials if they have fever, and so acquired immunity and, in particular, tolerance may be decreased.

If anti-disease immunity declines or at least does not increase with age, how then can the incidence of malaria morbidity also decline with age? Except in the case of naive individuals who have not developed tolerance, the main determinant of the age distribution of morbidity is the development of antiparasite immunity. Although recent studies of Javanese immigrants in Irian Jaya (not far from the Wosera study area) have suggested that such immunity can develop more rapidly in adults (Baird et al. 1991, 1993), the generally accepted view, which is consistent with the present study, is that antiparasite immunity builds up gradually with exposure, slowly restricting the density of asexual parasitaemia (McGregor \& Wilson, 1988). This may reflect either poor immunogenicity of the protective epitopes, or antigenic diversity and the consequent need for a long period in which to accumulate exposure to the whole repertoire of parasite strains circulating (Day \& Marsh, 1991). Our results are entirely consistent with such immunity resulting in a decrease in the risk of high-density infections in adults and consequently of morbidity. The very different age profiles for anti-parasite and antidisease immunity do not support the suggestion of Kwiatkowski (1991) that the latter represents incomplete anti-parasite immunity. These two components of the immune response to $P$. falciparum have very different age and exposure dependences.

The decline in tolerance with age is in agreement with the studies of Miller (1958) and Trape et al. (1985) who observed a decrease with age in the threshold parasite density leading to fever. A different conclusion was arrived at by Petersen et al. $(1991 a, b)$, who observed that at parasite isodensity, body temperatures show a steady decline with age, and interpreted this to mean that tolerance increases with age. However, both normal body temperatures (Kemp, Silver \& O'Brian, 1978) and the symptoms of malaria (Trape et al. 1985) are age dependent. Certain manifestations of malaria illness may become less severe with increasing age, but this does not contradict the finding that a pathological response is triggered at a lower parasite density threshold among older individuals. The clinical response to malaria infection involves various components of the immune system (McGregor \& Wilson, 1988) which evolve differently in response to exposure and to the ageing of the host, and this multiplicity of age and exposure dependences must be considered both in the design of vaccines and in the interpretation of clinical data from endemic areas.

We are grateful to residents of the Wosera area for their cooperation. We thank the staff at the Kunjingini Health Centre for their collaboration, and all those Institute of Medical Research staff involved in the fieldwork and microscopy. We would also like to thank Nicole Hurt for helpful discussions and comments on the manuscript. This work was part of the Malaria Vaccine Epidemiology and Evaluation Project funded by the United States Agency for International Development, No. 9365967.89.

\section{APPENDIX}

Let $\mathrm{P}(a, x)$ be the probability that an individual with parasitaemia $x$, in age group $a$, reports to the health facility over a given time-interval with a presumptive episode of malaria. From (2) above, the probability that the episode is $P$. falciparum-attributable is :

$\lambda(a, x)=\frac{R(a, x)-1}{R(a, x)}$

where $R(a, x)$ is the age-specific relative risk associated with parasitaemia $x$, which can be estimated from the logistic regression model (3).

Therefore the probability that such an individual reports with a $P$. falciparum-attributable episode is $\mathrm{P}(a, x) \lambda(a, x)$. But, from the definition of the relative risk,

$\mathrm{P}(a, x)=R(a, x) \mathrm{P}(a, 0)$

and hence:

$\mathrm{P}(a, x) \lambda(a, x)=(R(a, x)-1) \mathrm{P}(a, 0)$.

The probability that an individual reports with a non-malaria episode is assumed independent of parasitaemia, hence equal to $\mathrm{P}(a, 0)$, the probability of reporting if the individual is aparasitaemic. Thus, if $\mathrm{P}(a$,$) is the marginal probability of reporting for$ the age group and $\lambda(a$,$) is the corresponding$ summary attributable fraction:

$\mathrm{P}(a)=,\mathrm{P}(a, 0) /(1-\lambda(a)$,

From (A 3) and (A 4) it follows that:

$\mathrm{P}(a, x) \lambda(a, x)=\mathrm{P}(a),(R(a, x)-1)(1-\lambda(a)$,$) .$

Dividing the equivalent quantity for the standard age group and parasite density, this gives the following expression for the relative risk of reporting to the health facility with a $P$. falciparum-attributable episode $(r(a, x))$ :

$$
\begin{aligned}
r(a, x) & =\frac{\mathrm{P}(a, x) \lambda(a, x)}{\mathrm{P}\left(0,10^{4}\right) \lambda\left(0,10^{4}\right)} \\
& =\frac{\mathrm{P}(a,)(R(a, x)-1)(1-\lambda(a,))}{\mathrm{P}(0,)\left(R\left(0,10^{4}\right)-1\right)(1-\lambda(0,))}
\end{aligned}
$$

Let $N(a)$ be the number of individuals in the population in age group $a$ and $n(a)$ be the total 
number of presumptive cases in that age group. The age-specific relative risk of being a presumptive case is then:

$$
\mathrm{P}(a,) / \mathrm{P}(0,)=(n(a) N(0)) /(n(0) N(a)),
$$

and substituting this into (A 6), an expression which can be used to compute $r(a, x)$ is obtained:

$$
\begin{aligned}
r(a, x)= & \frac{\mathrm{P}(a, x) \lambda(a, x)}{\mathrm{P}\left(0,10^{4}\right) \lambda\left(0,10^{4}\right)} \\
& =\frac{n(a) N(0)(R(a, x)-1)(1-\lambda(a,))}{n(0) N(a)\left(R\left(0,10^{4}\right)-1\right)(1-\lambda(0,))} .
\end{aligned}
$$

\section{REFERENCES}

ALPERS, M. P., AL-YAMAN, F., BECK, H.-P., BHATIA, K., HII, J., LEWIS, D., PARU, R. \& SMITH, T. (1992). The malaria vaccine epidemiology and evaluation project of $\mathrm{PNG}$ : rationale and baseline studies. Papua New Guinea Medical fournal 35, 285-97.

ARMSTRONG SCHELLENBERG, J. R. M., SMITH, T., ALONSO, P. L. \& HAYES, R. J. (1994). What is clinical malaria? Finding case definitions for field research in highly endemic areas. Parasitology Today (in the Press).

BAIRD, J. K., PURNOMO, H. B., BANGS, M. J., ANDERSEN, E. M., JONES, T. R., MASBAR, S., HARJOSUWANO, S., Subianto, B. \& ARBani, P. R. (1993). Age-specific prevalence of Plasmodium falciparum among six populations with limited histories of exposure to endemic malaria. American Fournal of Tropical Medicine and Hygiene 49, 707-19.

BAIRD, J. K., TREVOR, R. J., DANUDIRGO, E. W., ANNIS, B. A., bangs, M. J., PURnomo, H. B. \& Masbar, s. (1991). Agedependent acquired protection against Plasmodium falciparum in people having two years exposure to hyperendemic malaria. American fournal of Tropical Medicine and Hygiene 45, 65-76.

BAte, C. A. W., TAVerne, J., DAVE, A. \& Playfair, J. H. L. (1990). Malaria exoantigens induce $T$-independent antibody that blocks their ability to induce TNF. Immunology 70, 315-20.

BRUZZI, P., GREEN, S., BYAR, D. P., BRINTON, L. A. \& SCHAIRER, C. (1985). Estimating the population attributable risk for multiple risk factors using casecontrol data. American fournal of Epidemiology 122, 904-14.

BURKot, T. R., GRAVES, P. M., CATTANi, J.A., WIRTZ, R. A. \& GIBSON, P. D. (1987). The efficiency of sporozoite transmission in the human malarias, Plasmodium falciparum and P. vivax. Bulletin of the World Health Organization 65, 375-80.

CATtaNi, J. A., TUlLoCh, J. L., VRBova, H., JOLley, D., GIBSON, F. D., MOIR, J. S., HEYWOOD, P. F., ALPERS, M. P., STEVENSON, A. \& CLANCY, R. (1986). The epidemiology of malaria in a population surrounding Madang, Papua New Guinea. American Yournal of Tropical Medicine and Hygiene 35, 3-15.

CHARLWOOD, J. D., GRAVES, P. M. \& ALPERS, M. P. (1986). The ecology of the Anopheles punctulatus group of mosquitoes from Papua New Guinea: a review of recent work. Papua New Guinea Medical Fournal 29, 19-26.
CHOUGNeT, C., DELORON, P. \& SAVEL, J. (1991).

Persistence of cellular and humoral response to synthetic peptides from defined Plasmodium falciparum antigens. Annals of Tropical Medicine and Parasitology 85, 357-63.

CLARK, I. A. \& CHAUDHRI, G. (1989). Relationships between inflammation and immunopathology of malaria. In Malaria : Host Responses to Infection (ed. Stevenson, M. M.), pp. 127-146. Boca Raton: CRC Press.

CoX, M. J., KUM, D., TAVUl, L., NARARa, A., RaIKo, A., BAISOR, M., ALPERS, M., MEDLEY, G. \& DAY, K. P. (1994). Dynamics of malaria parasitaemia associated with febrile illness in children from a rural area of Madang, Papua New Guinea. Transactions of the Royal Society of Tropical Medicine and Hygiene (in the Press).

DAY, K. P. \& MARSH, K. (1991). Naturally acquired immunity to Plasmodium falciparum. Immunology Today 7, A68-A71.

DELORON, P., LE BRAS, J., SAVEL, J. \& COULAUD, J. P. (1987). Antibodies to the PF155 antigen of Plasmodium falciparum: measurement by cell-ELISA and correlation with expected immune protection. American Yournal of Tropical Medicine and Hygiene 37, 22-6.

FELGER, I., TAVUl, L., KaBiNTIK, S., MaRShall, V., GENTON, B., ALPERS, M. \& BECK, H.-P. (1994). Plasmodium falciparum: extensive polymorphism in merozoite surface antigen 2 alleles in an area with endemic malaria in Papua New Guinea. Experimental Parasitology (in the Press).

GARNER, P. A. (1989). The Epidemiology of Maternal and Neonatal Health in Papua New Guinea. Ph.D. thesis. University of London.

GENTON, B., SMITH, T., BAEA, K., NARARA, A., AL-YAMAN, F., BECK, H.-P., HII, J. \& ALPERS, M. (1994). How useful are clinical criteria for improving the diagnosis of malaria among presumptive cases in a highly endemic area of Papua New Guinea? Transactions of the Royal Society of Tropical Medicine and Hygiene (in the Press).

GREENWOOD, B. M. (1987). Asymptomatic malaria infections - Do they matter? Parasitology Today 3 , 206-14.

GREENWOOD, B. M., BRADLEY, A. K., GREENWOOD, A. M., BYASS, P., JAMMEH, K., MARSH, K., TULLOCH, $\mathrm{S}$. OLDFIELD, F. S. J. \& HAYES, R. (1987). Mortality and morbidity from malaria among children in a rural area of The Gambia, West Africa. Transactions of the Royal Society of Tropical Medicine and Hygiene 81, 478-86.

KEMP, C. H., SILVER, H. K. \& O'BRIAN, D. (1978). Current Pediatric Diagnosis and Treatment, 5th Edn. Los Altos: Lange Medical Publications.

KWIATKowski, D. (1989). Febrile temperatures can synchronise the growth of Plasmodium falciparum in vitro. Fournal of Experimental Medicine 169, 357-61.

KWIATKOWSKI, D. (1991). Cytokines and anti-disease immunity to malaria. Research in Immunology 142, 707-12.

MCGREGOR, I. A. (1983). Current concepts concerning man's resistance of infection with malaria. Bulletin de la Société de Pathologie Exotique 76, 433-45. MCGREGOR, I. A. \& WILSON, R. J. M. (1988). Specific 
immunity: acquired in man. In Malaria, Principles and Practice of Malariology (ed. Wernsdorfer, W. H. \& McGregor, I.), pp. 559-620. Edinburgh : Churchill Livingstone.

MARSH, K. (1992). Malaria - a neglected disease? Parasitology 104, S53-S65.

MILLER, M. J. (1958). Observations of the natural history of malaria in the semi-resistant West African. Transactions of the Royal Society of Tropical Medicine and Hygiene 52, 152-68.

MOLINEAUX, L. (1988). The epidemiology of human malaria as an explanation of its distribution, including some implications for its control. In Malaria, Principles and Practice of Malariology (ed. Wernsdorfer, W. H. \& McGregor, I.), pp. 913-998. Edinburgh: Churchill Livingstone.

Olivar, M., DeVElouX, M., AGARI, A. C. \& LOUTAN, L. (1991). Presumptive diagnosis of malaria results in a significant risk of mistreatment of children in urban Sahel. Transactions of the Royal Society of Tropical Medicine and Hygiene 85, 729-30.

PAPUA NEW GUINEA DEPARTMENT OF HEALTH (1989). Standard Treatment for Common Illnesses of Adults in Papua New Guinea, 4th Edn. Port Moresby: Department of Health.

PETERSEN, E., HOGH, B., MARBIAH, N. T., DAVID, K. \& HANSON, A. P. (1991a). Development of immunity against Plasmodium falciparum malaria: clinical and parasitologic immunity cannot be separated. Fournal of Infectious Diseases 164, 949-53.

petersen, E., hogh, B., Marbiah, N. T., Dolopaie, E., GotTshau, A., hanson, A. P. \& BJORKMAN, A. (1991 $b$ ). Clinical and parasitological studies on malaria in Liberian adults living under intense malaria transmission. Annals of Tropical Medicine and Parasitology 85, 577-84.

Playfair, J. H. L., TAVERne, J., Bate, C. A. W. \& DE SOUZA, J. B. (1990). The malaria vaccine: anti-parasite or antidisease? Immunology Today 11, 25-7.

ROOTH, I. B. \& BJORKMAN, A. (1992). Suppression of Plasmodium falciparum infections during concomitant measles or influenza but not during pertussis.
American Yournal of Tropical Medicine and Hygiene 47, 675-81.

SELWYN, B. J. (1990). The epidemiology of acute respiratory tract infection in young children: comparison of findings from several developing countries. Reviews of Infectious Disease 12 (Suppl. 18), S870-S888.

SHUTE, G. T. (1988). The microscopic diagnosis of malaria. In Malaria, Principles and Practice of Malariology (ed. Wernsdorfer, W. H. \& McGregor, I.), pp. 781-814. Edinburgh: Churchill Livingstone. SMITH, T., ARMSTRONG SCHELLENBERG, J. R. M. \& HAYES, R. (1994). Attributable fraction estimates and case definitions for malaria in endemic areas. Statistics in Medicine (in the Press).

SMITH, T., LEHMANN, D., COAKLEY, C., SPOONER, v. \& ALPERS, M. P. (1991). Relationships between growth and acute lower respiratory infections among children under five years of age in a highland population of Papua New Guinea. American Journal of Clinical Nutrition 53, 963-70.

SNOW, R. W., LINDSAY, S. W., HAYES, R. J. \& GREENWOOD, B. M. (1988). Permethrin-treated bed nets (mosquito nets) prevent malaria in Gambian children.

Transactions of the Royal Society of Tropical Medicine and Hygiene 82, 838-42.

TRAPE, J. F., PEelman, P. \& MOZAult-PEelman, B. (1985). Criteria for diagnosing clinical malaria among a semiimmune population exposed to intense and perennial transmission. Transactions of the Royal Society of Tropical Medicine and Hygiene 79, 435-42.

TRAPE, J. F., ZOULANI, A. \& QUINET, M. C. (1987).

Assessment of the incidence and prevalence of clinical malaria in semi-immune children exposed to intense and perennial transmission. American fournal of Epidemiology 126, 193-201.

vince, J. D. (1992). Malaria in children in Papua New Guinea. Papua New Guinea Medical Fournal 35, 258-63.

WILSON, D. B., GARNHAM, P. C. C. \& SWELlENGREBEL, N. H. (1950). A review of hyperendemic malaria. Tropical Diseases Bulletin 47, 677-98. 